\title{
Human ESC: Model for brain development and repair
}

\author{
S-C Zhang ${ }^{1}$ \\ ${ }^{I}$ Departments of Anatomy and Neurology, School of Medicine and Public Health, Waisman Center, University of Wisconsin, \\ Madison, WI, USA
}

Human embryonic stem cells (hESCs), initiated from the inner cell mass of an early embryo, offer an unprecedented tool for unveiling normal and abnormal human development as well as developing medical therapeutics. We have established a chemically defined neural differentiation system that recapitulates most of the key events that occur during early human embryo development, such as formation of neural tube-like rosettes and appearance of region-specific progenitors at the correct time. With this model system we have discovered that the neuroectodermal differentiation from hESCs undergoes two morphologically and molecularly distinguishable stages, which we term the primitive and the definitive neuroectodermal cells. The uniform expression of anterior transcription factors in the primitive neuroepithelial cells, as opposed to the predominant $\mathrm{mid} / \mathrm{hind}$ brain neuroepithelial differentiation from mouse ESCs, reflects the need of building the large forebrain in primates. Our differentiation model hence sets a foundation for revealing genetic and epigenetic programs underlying the making of our brain.

The two-step neuroectodermal differentiation model lends a conceptual framework for correctly patterning the nalve neuroepithelial cells, thus leading to successful differentiation of forebrain glutaminergic and GABAergic neurons, midbrain dopamine neurons, spinal cholinergic motor neurons, and myelinating oligodendrocytes. Systematic electrophysiological and transplantation analyses indicate that neurons produced in a Petri dish function like authentic neurons, respond to the brain environment for maturation, and correct functional deficit in neurological animal models. The in vitro produced oligodendrocytes can produce myelin sheaths in the brain of myelin deficient animals. Thus, the in vitro generated neural cells may be useful for potential future cell therapy.

Cell Research (2008) 18:s172. doi: 10.1038/cr.2008.262; published online 4 August 2008

Correspondence: S-C Zhang

E-mail: zhang@waisman.wisc.edu

University of Wisconsin Madison

http://www.waisman.wisc.edu/scrp/zhang.html

Su-Chun Zhang is an associate professor of Anatomy and Neurology at the University of Wisconsin-Madison. He received his MD/MS at Shanghai Medical University (now Fudan University Shanghai Medical School) in China and his PhD training in Cell Biology in Canada. His research group investigates the neural differentiation pathways from hESCs. His team first established a model of neuroectoderm specification from $\mathrm{hESCs}$ in a defined culture system which resembles in vivo neural plate/tube development in humans. They have also successfully directed hESC-derived neuroectoderm to spinal motor neurons, midbrain dopamine neurons, and myelinating oligodendrocytes in chemically defined systems. Their current focus is to dissect genetic and epigenetic regulation of the differentiation processes from hESCs to neural subtypes, to create research and screening tools through genetic modification of hESCs, and to explore therapeutic potentials of hESC-derived neural cells in neurological rodent and primate models. (Supported by NIH [NS045926, NS046587, NS061243], ALS Association, Michael J. Fox Foundation, and National MS Society). 